\title{
Eficacia de la higiene oral en adultos jóvenes fumadores y no fumadores
}

\author{
Feser Gustavo*, Funosas Esteban*, Marí Gonzalo**, Escovich Livia***
}

\section{RESUMEN}

Para este estudio se escogió una muestra probabilística de una población de adultos jóvenes cursantes de cuarto año de la carrera de odontología de la Facultad de Odontología de Rosario, Argentina. Los objetivos fueron: determinar la prevalencia de fumadores en este grupo poblacional y luego establecer el grado de eficacia en la higiene oral llevada a cabo por este grupo de estudiantes. En una primera etapa, para determinar la prevalencia de fumadores se tomaron 150 alumnos de cuarto año y mediante un cuestionario se indago sobre las características del hábito de fumar, en lo referente a edad de iniciación y a la cantidad de cigarrillos consumidos por día. En una segunda etapa, para determinar la eficacia de las medidas de higiene oral, los alumnos fueron seleccionados al azar, totalizando 61 individuos, de los cuales 32 no poseían el hábito de fumar y 29 sí lo hacían. El promedio de la edad de los no fumadores fue de $23 \pm 2,109$ años, y en el grupo de fumadores de 23,655 $\pm 1,913$ años, no existiendo diferencia significativa. A cada individuo se le realizó el índice de higiene oral simplificado de Green - Vermillion con tinción. Todas las medidas fueron registradas por un examinador calibrado para evitar el sesgo interexaminador. El hábito de fumar fue cuestionado al final de la historia clínica para evitar la subjetividad del clínico. El índice de higiene oral asignado para cada grupo fue de 1,103 (IC 95\% 0,344-1,861), correspondiente a los fumadores y 1,218 (IC 95\% 0,245-2,191), correspondiente a los no fumadores. Con la confrontación de los datos y fijando el valor de p menor al $5 \%$ de error, concluimos que no hay diferencias significativas entre la acumulación de placa supragingival de uno y otro grupo y que la eficacia de la higiene oral entre adultos jóvenes fumadores y no fumadores estudiantes de odontología es semejante y aceptable. Este estudio sirve para determinar la prevalencia de fumadores en estudiantes del área de la salud y como avance para posteriores estudios de casos y controles que realizaremos en poblaciones semejantes, con el objeto de controlar la variable "higiene oral" como factor de confusión en el diseño etiológico causal que le corresponde al hábito de fumar.

Palabras clave: Fumar tabaco. Prevalencia. Higiene oral.

\section{SUMMARY}

This study was carried out on a population of young adult dentistry students. The objectives were to determine the prevalence of smokers in this group and to establish the effectiveness of oral hygiene practices of this group of students. In a first stage, a group of students were asked about their smoking habits, initiating age and amount of cigarettes per day, in order to determine the prevalence of smokers .In a second stage, students were randomized selecting a total of 61 individuals, 32 of them didn't have smoking habits and 29 has it; in order to determine oral hygiene's effectiveness. The age average of non smokers was $23 \pm 2,109$ years old, and of smokers $23,655 \pm 1,913$ year-old, not existing significant difference between them. Green $\&$ Vermillion simplified oral hygiene index was carried out to each student. All measures were registered by a gauged examiner to avoid the interexaminer mistake. Information on smoking habits was asked at the end of examination in order to avoid the subjectivity of the clinic examiner. The oral hygiene index assigned for each group was 1,103 (IC 95\% 0,344-1,861) for smokers and 1,218 (IC 95\% 0,245-2,191)for non smokers. By confronting data and fixing $\mathrm{p}$ value $<5 \%$, we conclude that there are no significant differences between the accumulation of supragingival plaque in both groups, and the effectiveness of oral hygiene among young adult smokers and non smokers is similar and acceptable. This study determines smokers prevalence in health-area students, and like an advance for future "casecontrol" studies that we'll carry out in similar populations, in order to control "oral hygiene" variable as a confusin factor in the etiologic causal design that corresponds to the smoking habit. 
Key words: Tobacco smoking. Prevalence. Oral higiene.

* Cátedra de Periodoncia. Facultad de Odontología.

** Escuela de Estadística. Facultad de Ciencias Económicas y Estadística.

**** Cátedras Estomatología I y II. Facultad de Odontología. Universidad Nacional de Rosario, Argentina

Feser Gustavo, Funosas Esteban, Marí Gonzalo, Escovich Livia. Eficacia de la higiene oral en adultos jóvenes fumadores y no fumadores. Av. Odontoestomatol 2003; 19-3: 141-148.

\section{INTRODUCCIÓN}

El tabaco y sus productos tóxicos están considerados como factores de riesgo para el hombre y responsables la de aparición de patologías tales como cáncer de pulmón, de boca, faringe y laringe, lesiones orales cancerizables y candidosis, entre otras (13). A su vez el tabaco está íntimamente ligado a una mayor prevalencia y severidad de periodontitis del adulto, periodontitis de establecimiento precoz, gingivitis úlcero-necrotizante aguda y periodontitis refractarias.

Los efectos tóxicos del tabaco producen una mayor pérdida dentaria en los individuos que poseen dicho hábito. Los fumadores tienen menos piezas dentarias que los no fumadores como lo demuestran algunos estudios epidemiológicos transversales y longitudinales (4-5). También se encuentra relacionado con la mayor destrucción de los tejidos periodontales y la aparición de patologías que responden menos a los tratamientos convencionales (6-13).

Algunos reportes epidemiológicos demostraron que los grandes fumadores (más de 20 cigarrillos diarios), tienen un ODDS RATIO de 5.0 a 7.0 para los casos diagnosticados de periodontitis severa (1416). Estudios recientes demostraron en poblaciones caucásicas de jóvenes con un promedio de edad de 19,38 $\pm 0,72$ años que el hábito de fumar determinaba una mayor profundidad de sondaje y mayor pérdida de inserción clínica (17). Otros estudios realizados sobre una extensa muestra poblacional de individuos adultos demostró que los grandes fumadores tenían mayor riesgo de pérdida de inserción periodontal (OR: 4,27) y pérdida ósea (OR: 7,28 IC $95 \% 5,09-10,31$ ) y que los fumadores leves (OR 2,05 para pérdida de inserción periodontal y OR: 3,25, IC $95 \%$ 2,33-4,54 para pérdida de tejido óseo) (18-19).
El consumo de tabaco puede realizarse de distintas formas: masticado en forma de chicles u hojas, aplicado localmente sobre la gíngiva en forma de un polvo finamente triturado o puede someterse a la quema mediante cigarros, cigarrillos rubios $\mathrm{y} / \mathrm{o}$ negros y pipas más la inhalación de su humo (20). Las formas de consumo varían según los países, las edades y los tiempos. También varía la prevalencia del hábito entre las distintas edades, países y estrato socio económico y cultural (21-23).

En Argentina también se observan variaciones de consumo y de prevalencia según las distintas zonas geográficas. Un estudio realizado en la ciudad de Rosario en el año 1991 (24), en una muestra de estudiantes de Medicina, de Ciencias Económicas y de jóvenes de la población general, demostró la prevalencia del hábito de fumar en éstos grupos poblacionales de diferentes condiciones sociales y económicas, y relacionó la presencia del hábito con otras variables estudiadas como son: edad de inicio, motivación de la iniciación y datos relevantes como la educación alcanzada y la ocupación de los padres. En aquel año este estudio determinó que la prevalencia de fumadores de una carrera relacionada con la salud era del $30 \%$ para los varones y de un $28 \%$ para las mujeres, porcentajes similares a los hallados en la población general.

Otro punto a tener en cuenta y que resulta conflictivo cuando se analizan otros reportes es la eficacia de la higiene oral entre los fumadores y los no fumadores. El problema principal se desprende en que la placa bacteriana o biofilm es también un factor de riesgo para enfermedad periodontal y en los estudios es imprescindible neutralizarla por la posibilidad de actuar como factor de confusión y con ello la posibilidad de una lectura errónea de los resultados obtenidos. Algunos reportes indican que los fumadores 
poseen una higiene oral deficiente y con ello más acumulación de placa y de cálculo que los no fumadores (25-34).

Otros estudios soportan la teoría que según las poblaciones elegidas los fumadores poseen una eficacia similar a los no fumadores en lo que se refiere al mantenimiento de una higiene oral adecuada, con esto neutralizarían el efecto de la placa bacteriana como factor de confusión (35-41).

Debido a la importancia que tiene el hábito de fumar sobre la salud periodontal, es importante conocer el comportamiento actual del hábito en poblaciones de adultos jóvenes que posibilite la confección de estudios epidemiológicos de mayor envergadura y la toma de decisiones a nivel sanitario.

\section{OBJETIVOS}

- Determinar la prevalencia de fumadores y no fumadores en adultos jóvenes cursantes de la carrera de Odontología en la Universidad Nacional de Rosario, Argentina.

- Determinar, mediante el uso de índices cuantificables de placa, la eficacia de la higiene oral de adultos jóvenes fumadores y no fumadores.

\section{MATERIALES Y MÉTODOS}

Para cumplir el primer objetivo se realizó un cuestionario a los alumnos de cuarto año de la carrera de Odontología. Se registraron las siguientes variables: la edad, el hábito de fumar o no hacerlo, la cantidad de cigarrillos por día consumidos y la edad de iniciación. La totalidad de alumnos encuestados fue 150.

Para el cumplimiento del segundo objetivo se decidió tomar una muestra de alumnos que fuera representativa y que permitiera el análisis posterior por métodos estadísticos. La muestra poblacional consistió de 61 alumnos cursantes de cuarto año de la carrera seleccionados al azar. Los motivos de exclusión fueron aquellos portadores de prótesis parcial removible o aparatología ortodóncica. A cada alumno se

le realizó el índice de placa de Greene y Vermillion (42), tiñendo los dientes representativos de cada arcada con fucsina básica en solución hidroalcohólica al $1 \%$. Los registros fueron tomados por el mismo examinador. Una vez tomado los índices se le cuestionó a cada individuo por el hábito de fumar o no hacerlo, lo que permitía incluirlo en uno u otro grupo de estudio y evitar así el sesgo de inclusión o de selección.

El valor de p se fijó con un error a menor a 0,05. Para comprobar la significación estadística se usó el test de Kolmogorov-Smirnov para dos muestras con distribución semejante

\section{RESULTADOS}

De los 150 alumnos encuestados, 94 (62,67\%) fueron mujeres y $56(37,33 \%)$ hombres (Tabla 1$)$. Con respecto al hábito de fumar, en las mujeres el $65 \%$ no posía el hábito y el $35 \%$ sí. En los hombres el hábito no estuvo presente en el $58 \%$ y sí lo estuvo en el $42 \%$ de los casos (Tabla 2). Por lo que se deduce que el hábito es ligeramente más prevalente en el hombre que en la mujer. Cuando analizamos la dosis diaria (número de cigarrillos consumidos por día) observa-

TABLA 1.- DISTRIBUICIÓN DEL GRUPO SEGÚN SEXO

\begin{tabular}{|lccc|}
\hline & $\begin{array}{c}\text { Frec. } \\
\text { absoluta }\end{array}$ & $\begin{array}{c}\text { Frec. } \\
\text { relativa }\end{array}$ & $\begin{array}{c}\text { Frec. } \\
\text { proporcional }\end{array}$ \\
\hline Mujeres & 94 & 0,63 & 62,67 \\
\hline Hombres & 56 & 0,37 & 37,33 \\
\hline Total & 150 & 1 & 100 \\
\hline
\end{tabular}

\section{TABLA 2.- DISTRIBUICIÓN DEL HÁBITO DE FUMAR SEGÚN SEXO}

\begin{tabular}{|lccc|}
\hline & No fuma & Fuma & Total \\
\hline Mujeres & $61(65 \%)$ & $33(35 \%)$ & 94 \\
\hline Hombres & $33(58 \%)$ & $23(42 \%)$ & 56 \\
\hline Total & $94(63 \%)$ & $56(37 \%)$ & 150 \\
\hline Mujeres & 0,41 & 0,22 & 0,63 \\
\hline Hombres & 0,22 & 0,15 & 0,37 \\
\hline Total & 0,63 & 0,37 & 1,00 \\
\hline
\end{tabular}




\begin{tabular}{|lccc|}
\hline \multicolumn{4}{|c|}{$\begin{array}{c}\text { TABLA 3.- DOSIS DE CIGARRILLOS } \\
\text { CONSUMIDA POR DÍA }\end{array}$} \\
\hline $\begin{array}{l}\text { No cigarrillos } \\
\text { por día }\end{array}$ & $\begin{array}{c}\text { Frec. } \\
\text { absoluta }\end{array}$ & $\begin{array}{c}\text { Frec. } \\
\text { relativa }\end{array}$ & $\begin{array}{c}\text { Frec. } \\
\text { proporcional }\end{array}$ \\
\hline de 1 a 10 & 32 & 0,57 & 57,14 \\
\hline de 10 a 20 & 20 & 0,36 & 35,71 \\
\hline más de 20 & 4 & 0,07 & 7,14 \\
\hline Total & 56 & 1,00 & 100,00 \\
\hline
\end{tabular}

\begin{tabular}{|lccc|}
\hline $\begin{array}{c}\text { TABLA 4. - EDAD DE INICIACIÓN DEL HÁBITO } \\
\text { DE FUMAR CIGARRILLOS }\end{array}$ \\
\hline $\begin{array}{c}\text { Frec. } \\
\text { absoluta }\end{array}$ & $\begin{array}{c}\text { Frec. } \\
\text { relativa }\end{array}$ & $\begin{array}{c}\text { Frec. } \\
\text { proporcional }\end{array}$ \\
\hline menor de 13 & 4 & 0,07 & 7,14 \\
\hline de 13 a 14 & 12 & 0,21 & 21,43 \\
\hline de 15 a 16 & 14 & 0,25 & 25,00 \\
\hline mayor de 16 & 26 & 0,46 & 46,43 \\
\hline Total & 56 & 1,00 & 100,00 \\
\hline
\end{tabular}

mos que el 57,14 \% eran fumadores leves, $(<10$ cigarrillos/día), el 35,71 \% fumaba de 10 a 20 cigarrillos diarios y sólo el $7,14 \%$ fumaba más de 20 cigarrillos al día ("heavy smokers" o grandes fumadores). Si analizamos la edad de iniciación del hábito, el $46,43 \%$ empezó a fumar después de los 16 años de edad, el mismo porcentaje (46,43\%), inició el hábito entre los 13 y los 16 años y sólo el 7,14 \% comenzó a fumar antes de los 13 años de edad.

El índice de higiene oral de Green y Vermillion fue 1,103 (IC 95\% 0,344-1,861) para los fumadores y 1,218 (IC 95\% 0,245-2,191) para los no fumadores. Mediante el análisis estadístico se demostró que las diferencias en la higiene encontradas en ambos grupos no son estadísticamente significativas; por lo tanto, con este estudio concluimos que no hay diferencias entre la higiene oral de adultos jóvenes, fumadores y no fumadores, y que el nivel alcanzado es aceptable para ambos grupos.

\section{DISCUSIÓN}

Los efectos deletéreos del cigarrillo y sus más de 3000 productos químicos tóxicos inducen cambios a nivel oral y a nivel sistémico, especialmente de tipo inmunológico (43-49). Además de las distintas lesiones que puede provocar en la cavidad bucal como cáncer de boca, lesiones micóticas y leucoplasias, entre otras, el tabaco es causa importante de lesiones en vasos sanguíneos, corazón, laringe, faringe y pulmones. Está altamente ligado a las enfermedades periodontales, como periodontitis refractarias, gingivitis ulcero necrotizante aguda y a la pérdida de tejido óseo y dentario (50-53).

Diversos estudios epidemiológicos de distintos diseños han tratado de dilucidar la problemática del consumo de tabaco. Incluso la Organización Mundial de la Salud, lo designó como un "verdadero problema de salud pública en diferentes países".

La encuesta realizada en éste estudio llega a la conclusión que hay un aumento en la prevalencia de fumadores en jóvenes universitarios, realizando una comparación con un estudio realizado en la ciudad de Rosario en 1991 sobre distintos grupos de jóvenes: población general, estudiantes de la carrera de Ciencias Económicas y un tercer grupo correspondiente a estudiantes de la carrera de Medicina (24). Si tomamos este último grupo, con un promedio de edad de 20 años, la prevalencia del hábito es de $30 \%$ para los varones y del $28 \%$ para las mujeres. Estas cifras difieren de las obtenidas en nuestro estudio, siendo los valores de $42 \%$ de fumadores varones y del $35 \%$ de fumadoras mujeres, con un promedio de edad de 23 años. Esto podría indicar que en diez años se produjo un crecimiento en la prevalencia del hábito en jóvenes estudiantes de carreras universitarias relacionadas con la salud y con características sociales y culturales semejantes, pertenecientes a la ciudad de Rosario, Argentina. Otros estudios muestran cifras diferentes de prevalencia del hábito tabáquico en jóvenes, que varían según los distintos países: $75,9 \%$ (54), $53 \%$ (55) y $24,5 \%$ (56).

Nuestros resultados con respecto a la edad de iniciación difieren de los resultados obtenidos por otras investigaciones, donde la edad de iniciación de los jóvenes incluidos dentro del estudio fue de 13,6 años tanto para hombres como para mujeres (57). En nuestro estudio las cifras mostraron los siguientes valores: el 7,14\% empezó antes de los 13 años, el $21,43 \%$ empezó entre los 13 y 14 años, el $25 \%$ se ini- 
ció entre los 15 y 16 años y el 46,43\% lo hizo después de los 16 años de edad, por lo que la exposición de éste grupo a los efectos perjudiciales del tabaco, sería menor que otros grupos de jóvenes.

Algunas publicaciones consideran que el hábito de fumar cigarrillos rubios ha declinado en las pasadas dos décadas para dar lugar a otro tipo de modalidad, como es el consumo de tabaco en forma de una mezcla finamente procesada para ser colocada localmente en las regiones gingivales de la cavidad bucal. Ésta adicción sería más prevalente entre los adolescentes varones (58), pero esta modalidad no se ha difundido en nuestro país.

Con respecto al mantenimiento de la higiene oral, los resultados de esta investigación difieren de trabajos realizados en otros países. Éstos informes determinaron para los fumadores una tasa de formación mayor de placa bacteriana y de cálculo, concluyendo que los que fumaban poseían una higiene oral deficiente (25-34). Nuestros resultados son coincidentes con otra investigaciones, donde el estándar de higiene oral fue hallado aceptable para grupos de no fumadores y fumadores (35-41). Los fumadores obtuvieron un índice de higiene oral semejante y sin diferencias estadísticamente significativas que los no fumadores. La semejanza en ésta variable, nos permite trabajar sobre grupos donde el factor "higiene oral" se encuentra neutralizado como elemento de confusión y así poder determinar la relación causal entre tabaco y enfermedad periodontal en adultos jóvenes.

\section{CONCLUSIONES}

Se obtuvo un índice de higiene oral semejante y sin diferencias estadísticamente significativas entre los fumadores y los no fumadores. La semejanza en ésta variable, nos permite trabajar sobre grupos donde el factor "higiene oral" se encuentra neutralizado como elemento de confusión y así poder determinar la relación causal entre tabaco y enfermedad periodontal en los adultos jóvenes.

La utilidad de ésta investigación es proveer conocimiento sobre el comportamiento del hábito de fumar y su progresión lineal con respecto a su prevalencia en un grupo de estudiantes universitarios de la ciudad de Rosario. También provee información para el desarrollo de estudios de casos y controles y transversales, que ayuden a la valoración del riesgo de fumar con respecto a la salud del periodoncio de inserción en adultos jóvenes y a la posibilidad de la toma de decisiones desde el área de salud para la educación y la disminución del hábito de fumar que hoy se encuentra en aumento entre los jóvenes de nuestra ciudad.

\section{AGRADECIMIENTOS}

A Mariana Gulino, Carolina González y Natalia Bellotto por su desinteresada colaboración en el presente trabajo.

\section{BIBLIOGRAFÍA}

1. Peto R, López AD, Boreham J, Thun M, Heath C, Jr. Mortality from tobacco in developed countries: indirect estimations from national vital statistics. Lancet 1992; 339: 1269-78.

2. US Departament of Health and Human Services. The health consequences of smoking: Cancer. Areport of Surgeon-General. USDHHS, Public Health Service, Centres for Disease Control Office on Smoking and Health, 1982; DHHS publications $\mathrm{n}^{\circ}$ (CDC) 82-50179.

3. US Departament and Health and Human Services. The Health Consequences of smoking: Cardiovascular disease. Areport of surgeongeneral. USDHHS, Public Health Service, Centres for disease Control Office on Smokingand Health, 1983; DHHS publications $\mathrm{n}^{\circ}$ (CDC) 84-50204.

4. Ragnarsson E, Aliasson S, Olafsson S. Tobacco smoking, a factor in tooth loss in Raykjavit, Iceland. Scand J Dent Res 1992; 100: 322-6.

5. Bolin A, Eklund G, Frithiof L, Lavstedt S. The effect of changed smoking habits on marginal alveolar bone loss. Swed Dent J 1993; 17: 2116. 
6. Preber H, Bergström J. Cigarette smoking in patients referred for periodontal treatment. Scan J Dent Res 1986; 94: 34-9.

7. Lavstedt S. A methodological-roentgenological investigation on marginal alveolar bone loss. Acta Odontol Scand 1975; 33 (Suppl. 67): 5087.

8. FeldmanRS, Bravacos JS, Rose CL. Association between smoking different tobacco products and periodontal disease indexes. J Periodontol 1983; 54: 481-7.

9. Bergström J, Eliasson S. Noxious effect of cigarette smoking on periodontal health. J Periodontol Res 1987; 2: 513-7.

10. Goultschin J, Sgan Cohen HD, Donchin M, Brayer L, Soskolne WA. Association of smoking with periodontal treatment needs. J Periodontol 1990; 61; 364-7.

11. Haber J, Kent RL. Cigarette smoking in a periodontal practice. J Periodontol 1992; 63; 100 106.

12. Magnusson I, Walker CB. Refractory periodontitis or recurrence of disease. J Clin Periodontol 1996; 23; 289-92.

13. Martinez-Canut P, Lorca A, Magan R. Smoking and periodontal disease severity. $\mathrm{J}$ Clin Periodontol 1996; 22: 743-9.

14. Dunford RJ, Lyon E, Ho Aw, et al. Smoking as a significant risk factor for severe periodontal adult disease. J Dent Res 1991; 70 : 590 (Abstr. 857).

15. Stoltenberg IL, Osborn JB, Hardie NA, Herzberg MC, Philstrom BL. The association between periodontal status and cigarette smoking. J Dent Res 1992: 70: 556 (Abstr. 2321).

16. Haber J, Wattles J, Crowby M, Mandell R, Kaunudi J, Kent R. Evidence for a cigarette smoking as a major risk factor for periodontitis.J Periodontol 1993; 63: 16-23.
17. Machuca G, Rosales I, Lacalle J, Machuca C, Bullón P. Effect of cigarettes smoking on periodontal status of healthy young adults. J Periodontol 2000; 71: 73-8.

18. Grossi SG, Zambon JJ, Ho AW, Koch G, Dunford RG, Matchei EE. Assessment of risk fpr periodontal disease. I. Risk indicators for attachment loss. J Periodontol 1994; 65 (3): 260-7.

19. Grossi SG, Genco RJ, Matchei EE, et al. Assessment of risk for periodontal disease. II. Risk indicators for alveolar bone loss. J Periodontol 1995; 66 (1): 23-9.

20. Poore TK, Johnson GK, Reinhardt RA, Organ CC. The effect of smokeless tobacco on clinical parameters of inflammation and gingival crevicular fluid prostaglandin E2, Interleukin $-1 \alpha$, and Interleukin -1ß. J Periodontol 1995; 66: 177-83.

21. Ernster, V. Trends in smoking, cancer risk and cigarette promotion. Cancer 1988; 62 (Suppl): 1702-12.

22. Mc Nelly, AD, et al. Prospective study of factors predicting uptake of smoking in adolescents. J Epidem Community Hlth. 1989; 43: 519-26.

23. Rogers RS, Grank J. Ethnic differences in smoking patterns: findings from NHIS, Publ Hlth Rep. 1988; 103: 387-93.

24. Poletto L, Pezzotto SM, Morini J, Andrade J. Prevalencia del hábito de fumar en jóvenes y sus padres. Asociaciones relevantes con educación y ocupación. Rev. Saúde públ. S.Paulo 1991;25 (5): 388-393.

25. Preber H, Kant T. Effect of tobacco-smoking on periodontal tissue of 15-years-old schoolchildren. J Periodont Res 1973; 8: 278-83.

26. Ainamo J. The seeming effect of tobacco consumption on the ocurrence of periodontal disease and dental caries. Suomen Hammaslaaskariseuran Toimituksia 1971; 67: 87-94.

27. Alexander AG. The relationship between tobacco 
smoking calculus and plaque accunulation and gingivitis. Dental health 1970; 9: 6-9.

28. Macgregor IDM. Toothbrushing efficiency in smokers and non.smokers. J Clin Periodontol 1984; 11: 313-20.

29. Arno A, Schei O, Lovdal A, Warehaug J. Alveolar bone loss as a function of tobacco consumption. Act Od Scand 1959; 17: 3-9.

30. Bergström J, Floderus-Myrhed B. Co-twin control study of the relationship between smoking and some periodontal disease factors. Community Dentistry and Oral Epidemiology 1983; 11: 113-6.

31. Feldman RS, Bravacos JS, Rose CL. Association between smoking different tobacco products and periodontal disease indexes. J Periodontol 1983; 54: 481-8.

32. Lavstedt S. A methodological-roengenological investigation on marginal alveolar bone loss. Thesis Acta Od Scand 1975; 33, Suppl 67.

33. Sheiham A. Periodontal disease and oral cleanliness in tobaccco smokers. J Periodontol 1971; 42: 259-63.

34. Gunsolley JC, Quinn SM, Tew J, Gooss CM, Brooks CN, Scenkein HA. The effect of smoking on individuals with minimal periodontal disease. J Periodontol 1998; 69:'165-70.

35. Bergström J, Eliasson S. Cigarette smoking and alveolar bone height in subjects with a high standard of oral higiene. J Clin Periodontol 1987; 14: 466-9.

36. Bergström J, Eliasson S. Noxious effect of cigarette smoking on periodontal health. J Periodontol Res 1987; 22: 513-7.

37. Danielsen B, Manji F, Nagerkerke N, Fejerskov O, Baelum V. Effect of cigarette smoking on the transition dynamics in experimental gingivitis. J Clin Periodontol 1990; 17: 159-64.
38. Bergström J. Oral higiene compliance and gingivitis expresión in cigarette smoking. Scan J Dent Res 1990; 98: 497-503.

39. Bergström J, Eliasson S, Preber H. Cigarette smoking and periodontal bone loss. J Periodontol 1991; 62: 242-6.

40. Kerdvongbundit V, Wikesjö UME. Effect of smoking on periodontal health in molar teeth. $\mathrm{J}$ Periodontol 2000; 71: 433-7.

41. Mullaly BH, Linden GJ. Molar furcation involvement associated with cigarette smoking in periodontal referrals. J Clin Periodontol 1996; 23: 658-61.

42. Greene JC, Vermillion JR. The simplified oral higiene index. J Am Dent Assoc 1964; 68: 7-13.

43. American Academy of Periodontology. Tobacco use and the periodontal patient (position paper). J Periodontol 1996; 67: 51-6.

44. Raulin LA, McPherson JC, McQuade MJ, Hanson BS. The effect of nicotine on the attachment of human fibroblasts to glass and human root surfaces in vivo. J Periodontol 1988;59:318-25.

45. Kenney EB, Kraal JH, Saxe SR, Jones J. The effect of cigarette smoke on human oral polymorphonuclear leukocytes. J Periodont Res 1977; 12: 227-34.

46. Kraal JH, Chancellor MB, Bridges RB, Bemis KG, Hawke JE. Variations in the gingival polymorphonuclear leukocyte migration rate in dogs induced by chemotactic autologous serum and migration inhibitor from tabacco smoke. J Periodont Res 1977; 12: 242-9.

47. Holt PG. Inmune and inflamatory function in cigarette smokers. Thorax 1987; 42: 241-9.

48. Jonson JD, Houchens DP, Kluwe WM, Craig DK, Fisher GL. Effects of mainstream and environmental tobacco smoke on the inmune system in animals and humans. A rewiew. Crit Rev Toxicol 1990; 20: 369-95. 
49. Clarke NG, Shepard BC, Hirsch RS. The effect of intra-arterial epinephrine and nicotine on gingival circulation. Oral Surg Oral Med Oral Pathol 1981; 52: 577-82.

50. Christen AG, Mc Donald Jl Jr, Christen Ja. The impact of tobacco use and cessation on nonmalignant and precancerous oral and dental disease and conditions. Indianápolis: Indiana University School of Dentristy 1991: 1-2.

51. Mecklenburg RE, Greenspan D, Manley MW. Tobacco effect in the mouth. Bethesda, Md: $\mathrm{NIH}$ publication $n^{\circ}$ 93-3330; 1991: 5-13.

52. Palmer RM. Tobacco smoking and oral health. Health Education Authority. Ocasional paper $n^{\circ}$. 6; 1987.

53. Tobacco production. Enciclopedia Britannica. Chicago: 1981; 18: 464-7.

54. Hashim R, Thomson WM, Pack ARC. Smoking in adolescence as a predictor of early loss of periodontal attachment. Community Dent Oral Epidemiol 2001; 29: 130-5.
55. Machuca G, Rosales I, Lacalle JR, Machuca C, Bullón P. Effect of cigarette smoking on periodontal status of healthy young adults. J Periodontol 2000; 71: 73-8.

56. López R, Fernández O, Jara G, Baelum V. Epidemiology of clinical attachment loss in adolescents. J Periodontol 2001; 72: 1666-74.

57. Preber H, Kant T. Effect of tobacco-smoking on periodontal tissue of 15-years-old schoolchildren. J Periodont Res 1973; 8: 278-83.

58. Poore TK, Johnson GK, Reinhardt RA, Organ CC. The effect of smokeless tobacco on clinical parameters of inflammation and gingival crevicular fluid prostaglandin E2, Interleukin $-1 \alpha$, and Interleukin -1ß. J Periodontol 1995; 66: 177-83.

\section{CORRESPONDENCIA}

Dra. Escovich

Entre Rios 2528

2000-Rosarioo

Argentina 